\title{
Referentes formativo-deportivos en el fútbol base español: un escenario socioeducativo complejo
}

The formatives and sports references in Spanish school football: A socioeducative complex framework.

\author{
Abel Merino Orozco \\ Universidad de Zaragoza, España \\ abmerino@unizar.es \\ Laurane Jarie \\ Universidad de Zaragoza, España \\ lauranejarie@msn.com \\ Pablo Usán Supervia Universidad \\ de Zaragoza, España \\ pablousan@hotmail.com
}

\section{Resumen:}

El fútbol es la actividad físico-deportiva más practicada en España en edad escolar y posee implicaciones formativas de relevancia para quien lo practica. Existe un interés educativo por intervenir en el deporte a fin de revalorizar las oportunidades formativas únicas que este ofrece. Por ello, se han emprendido acciones que inciden en pautar el comportamiento de formadores y familiares. Este artículo pretende realizar un recorrido exhaustivo por los referentes formativos que contribuyen en los aprendizajes que los niños construyen, desde la premisa de que la no formalidad del escenario facilita la permeabilidad de referentes dando lugar a un espacio dinámico y complejo. Esta percepción pretende favorecer una certidumbre formativa que vincule la convergencia de las buenas prácticas que atiendan la complejidad referencial del escenario formativo-deportivo que es el fútbol escolar.

Palabras Clave: Formación deportiva, Fútbol, Deporte escolar, Familia, Entrenador.

\section{Abstract:}

Football is the sport most practiced in Spain in school age and it has formative implications of relevance for those who practice it. There is an educational interest in intervening in sport in order to revalue the unique training opportunities it offers. Therefore, actions that involve the behavior of trainers and family members have been undertaken. This article intends to make an exhaustive route of the formative referents that contribute to the learning that the children construct. The non-formality of the scenario facilitates the permeability of referents, which gives rise to a dynamic and complex framework. This perception aims to create formative certainty linking the converging points of good practices, which take into account the referential complexity of the formative-sport framework: the school football.

KEYWORDS: Sport training, Football, School Sport, Family, Coach.

\section{INTRODUCCIÓN}

Este artículo supone una revisión de la literatura científica en el ámbito formativo-deportivo del fútbol base. El foco reside en no tanto la adaptación del deporte al niño, sino la comprensión del entorno socioeducativo como base del aprendizaje del niño a fin de explorar su potencial formativo. Castejón (2008) se refiere a la particularidad de este escenario socioeducativo debido a la gran afluencia de familiares que arengan por el simple hecho de que son sus hijos quienes están jugando y, todo ello, generalmente coordinado por un entrenador sin una formación pedagógica específica para potenciar el valor formativo del deporte. Realidad 
absolutamente palpable, apostilla, si un sábado por la mañana uno pasea por algún colegio cercano a la propia residencia y que da lugar a una imagen absolutamente 'deporte-centrista' ${ }^{1}$.

La primera consideración que se ha de tener en cuenta es que el fútbol base en España se integra dentro del deporte escolar, por lo que el estado cede las competencias para la organización y gestión de las competiciones a cada Comunidad Autónoma, no existiendo uniformidad en los planes y programas desarrollados por cada región para la edad escolar (Arufe-Giráldez, Barcala-Furelos \& Mateos Padorno, 2017). A su vez, se ceden las competencias sobre la gestión económica del deporte escolar a las provincias que integran las Comunidades Autónomas, las federaciones deportivas, y a los organizadores: bien sean ayuntamientos, entidades deportivas, asociaciones privadas etc. (Ibáñez, del Barrio, Gómez \& Araujo, 2007). Si bien, estas apenas han dedicado esfuerzos convergentes en la formalización de una reglamentación común o, simplemente, la construcción de un reglamento consolidado, sino que se circunscribe a la organización espacio-temporal de la actividad. Esta divergencia favorece que se emprendan propuestas formativo-deportivas diferentes, pero con puntos convergentes y transferibles entre sí. Este documento pretende realizar un recorrido por la fundamentación teórica que justifica la intervención en el fútbol base a fin de esbozar los elementos a considerar para abordar el deporte y revalorizar su sentido formativo (Merino, Arraiz \& Sabirón, 2017a).

\section{COMPRESIÓN DEL ENTRAMADO INTERPERSONAL SOCIOCONSTRUIDO}

La literatura científica ha focalizado la atención en las relaciones que se consideran nucleares o vertebradores en el fútbol formativo, de modo que se fragmenta disciplinalmente la realidad para su estudio (Merino, Arraiz \& Sabirón, 2015). Aquí, se pretende abordar una comprensión de una realidad compleja, un tejido conjunto; sin embargo, a fin de emprender la comprensión, se analiza la vertebración que tradicionalmente ha explicado el escenario. En primer lugar, se han invertido esfuerzos por entender la relación paterno-filial y en el estudio de la figura del formador. Posteriormente, se ha definido e intervenido sobre el esqueleto formado por padres, formadores y jugadores. Finalmente, se ha puesto el foco sobre otros referentes que habrían de considerarse.

La permeabilidad del escenario facilita que exista mayor comunicación entre los participantes en el fútbol correspondiente a los 7 y 8 años que en otros deportes escolares (Garrido, González \& Romero, 2010). Cara, Pernía y Utrilla (2014) defienden la necesidad de que el entorno adulto, compuesto por padres, entrenador y árbitro, coopere para ofrecer una formación deportiva coherente, especialmente entre formadores y padres, ya que existe un interés explícito por potenciar el progreso del niño. Así, se ha de favorecer un entramado social adulto que revalorice la labor del formador, que con sus acciones genera las consecuencias formativas sobre el jugador que pueden trascender a los apriorísticos intereses formativos (Abós, Sevil, Sanz, Aibar y García, 2015).

\section{Relación PATERno-FiLial}

Uno de los referentes fundamentales para los niños de seis y siete años son los padres, por ello requiere un análisis especial; de hecho, en estas edades son ellos quienes promueven su prosocialidad, responsabilidad y autogestión mediante su estilo educativo y la dinamización de diferentes prácticas deportivas (Suldo, 2009; Ossorio, 2012). Particularmente, existe una focalización tanto en las creencias sobre el significado del fútbol escolar que los padres trasmiten a sus hijos como sus comportamientos reales. Como primeros agentes educativos poseen una responsabilidad primitiva en la educación de sus hijos, por lo que se parte del axioma de que la inversión de tiempos y esfuerzos que realizan en el acompañamiento de la actividad físico-deportiva de sus niños implica una motivación inherente hacia una formación de calidad para ellos (Merino, Arraiz \& Sabirón, 2016). 
Se ha de tener en cuenta que la relación paterno-filial tiene trascendencia mucho más allá de los límites temporales del escenario objeto de estudio; por lo tanto, repercute determinantemente en el valor educativo atribuido al escenario. La ejemplificación a través de las actitudes y comportamientos tiene un valor específico, ya que diversos estudios demuestran que es significativamente inferior el abandono de la actividad física en niños cuyas familias practican que en las que no lo hacen (Anderssen \& Wold, 1992; Isorna, Ruiz \& Rial, 2013; Piéron, 2002; Raudsepp \& Viira, 2000; Romero, Garrido \& Zagalaz, 2009). El interés para Reschly y Christenson (2009) reside en la asociación del entorno adulto para que el mensaje que se trasmita a los niños sea, como mínimo, congruente, responsable y, consecuentemente, comprometido con los valores educativos. Por lo que se apunta a una necesidad de coordinación interpersonal adulta.

Los padres tienen un valor añadido con su actitud en el escenario, pues es ejemplarizante desde la paradoja entre las expectativas y creencias que depositan en el fútbol formativo y los comportamientos espontáneos que pueden evidenciar en los campos de fútbol dejándose llevar por la emoción competitiva del momento (Merino, Arraiz \& Sabirón, 2017b). Más allá, Suldo (2009) considera que los padres han de acompañar a sus hijos a la consecución de metas difíciles pero coherentes con los valores que se desean trasmitir y adecuadas a sus posibilidades, de forma que se favorezca la autoeficacia. Igualmente se tiene en cuenta el potencial del aprendizaje por imitación del referente paterno, que es ejemplarizante. López (2005, p. 5) señala la importancia de que las figuras paternas no traten de llegar a controlar cada conducta de su hijo, sino que meramente han de garantizar si "un entorno rico y variado, gratificante, que permita al alumno enfrentarse a diferentes situaciones y superarlas, ayudando a desarrollar su autoestima y el conocimiento de sí mismo”. Por tanto, para comprender el escenario es indispensable aludir a la relación paterno-filial.

A las familias se les atribuye en papel indispensable en la educación de sus niños. En su tiempo de ocio, invierten sus esfuerzos en facilitar las actividades extraescolares con la convicción de que, así, facilitan su educación integral. Sus intenciones son plenamente constructivas, pero, en ocasiones, demandan cierto asesoramiento sobre cómo podrían optimizar su comportamiento para contribuir positivamente con los valores y competencias promovidas en los escenarios formativos, pues son conscientes que, en ocasiones, pueden actuar involuntariamente en perjuicio de sus hijos (Buceta, 2015; Merino, Arraiz \& Sabirón, 2016). Los familiares no habrían de ser el enemigo, sino, aprovechando la permeabilidad del escenario, un aliado que aporte coherencia al sentido educativo del deporte escolar. En la figura 1 se sintetizan las responsabilidades derivadas de la implicación familiar en el escenario.

\section{FIGURA 1}

Síntesis de la relevancia educativa del familiar en el deporte escolar

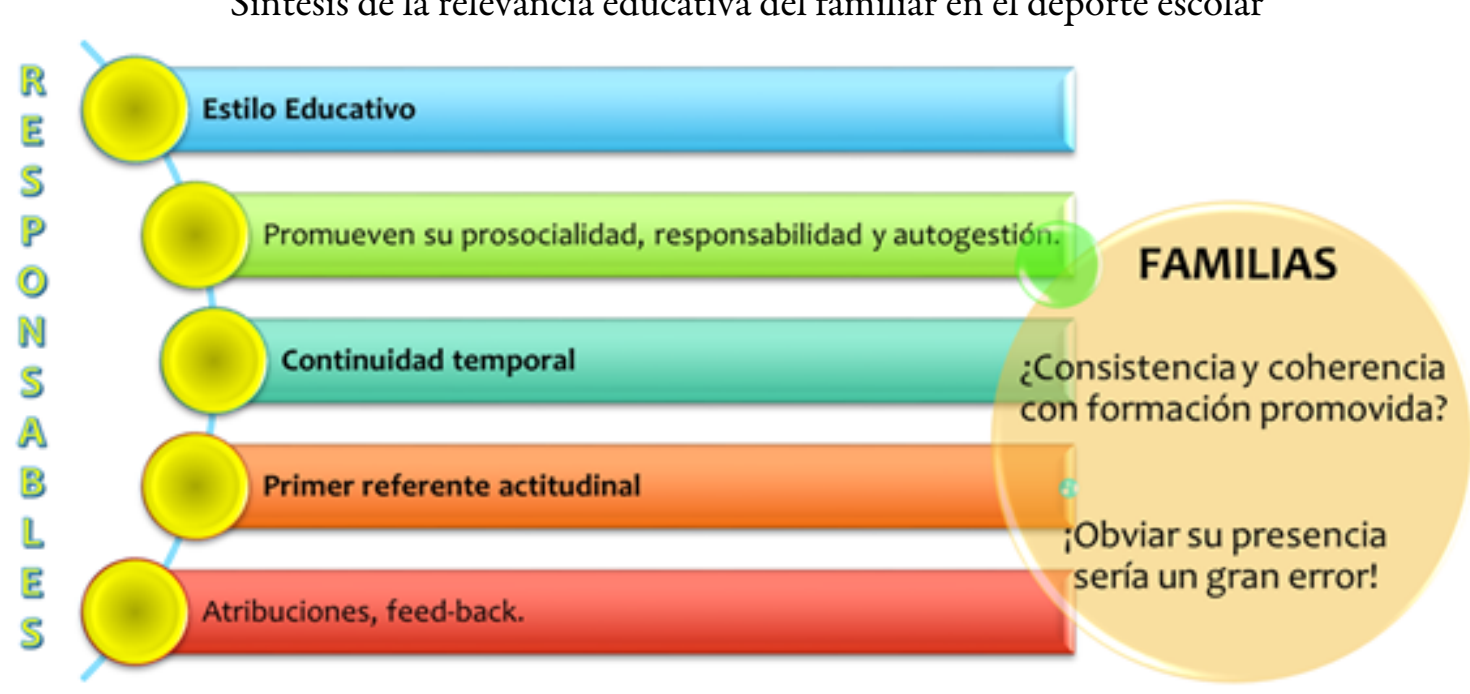

Fuente: elaboración propia. 


\section{EL FORMADOR}

El formador deportivo o entrenador de fútbol, en este caso, emerge como figura clave en la coordinación del rumbo formativo-deportivo que adquiere el equipo del fútbol. Sobre su figura recaen responsabilidades y presiones derivadas desde el club al que pertenece, las familias que confían la custodia y educación de su bien más preciado, de las demandas del deporte de competición y de las administraciones que organizan la competición reglada. Como norma general, sin una remuneración económica profesional formalizada que compense su responsabilidad (Merino, Arraiz \& Sabirón, 2017b).

Para París (2015), esta circunstancia no pasa desapercibida, puesto que ciertas preocupaciones por el deporte escolar en cuanto a los riegos de sedentarismo, abandono, primacía de la competición y sesgo por género, que son manifiestas en España, son compartidas por instancias europeas, por lo que se están promoviendo acciones que faciliten el empoderamiento de los encargados de gestionar el deporte escolar. Este interés tiene réplica en la concreción del fútbol base, ya que la figura del entrenador de fútbol base está siendo objeto de estudio emergente como clave en la formación del joven deportista (Gilbert \& Rangeon, 2011; Merino, Arraiz \& Sabirón, 2017b). La convergencia de esfuerzos en la comprensión de las dificultades desde la perspectiva del formador, en un ámbito no formal, abre una prospectiva a la mejora de las posibilidades educativas que ofrece el deporte escolar como complemento a la educación formal. Además, supone una revalorización de su labor, pues implica el reconocimiento de que el formador deportivo no es un mero instructor de conocimientos futbolísticos, sino que se contempla como profesional encargado de la coordinación de un complejo escenario en el que el protagonista es el deportista, a quien se le proporciona un proceso de acompañamiento formativo-deportivo (Gimeno, 2000; Merino, Sabirón \& Arraiz, 2015; Monjas, Ponce \& Gea, 2015). En Norteamérica se sintetiza este agente en la figura del coach, como acompañante transdisciplinar del jugador, de quien se anhela y reivindica su profesionalización (Duffy, et al., 2011).

Abad, Giménez, Robles y Rodríguez (2011) han analizado la paradójica situación de los entrenadores en el fútbol base de la provincia de Huelva. En su estudio, que indaga sobre su perfil y experiencia, se encuentra que es frecuente que sean jóvenes varones de entre 21 y 30 años que han practicado o practican fútbol y con una experiencia de entre cero y cinco años en el fútbol base como formadores. Además, se concluye que los formadores participantes en el mismo poseían escasos conocimientos pedagógicos y que la mayoría utilizan métodos eminentemente instructivos. Estos resultados subrayan el perfil tradicional al que se ha asociado al entrenador de fútbol base: un entrenador con escasa formación reglada, especialmente en el ámbito pedagógico y deportivo (Nuviala, León, Gálvez \& Fernández, 2007; Castejón, 2008; González, 2012; Monjas, Ponce \& Gea, 2015). Al respecto, un estudio con entrenadores de jugadores en etapa escolar indica que una mayor formación pedagógica formal en el área de la Educación Física redunda positivamente en que se utilicen metodologías que respetan los principios pedagógicos actuales del deporte escolar (Lledó, Martínez \& Huertas, 2014), mientras que la menor experiencia como entrenador y jugador se correlaciona con una menor aplicación práctica de los factores de eficacia en el fútbol (Duarte, Garganta \& Ferreira, 2014). Además, padres y deportistas confluyen en que un buen entrenador ha de definirse por ser "buen comunicador, que enseñe, que escuche y que trasmita confianza” (Gimeno, 2000, p. 11). Llopis (2011) indica que un entrenador no sólo ha de evidenciar cualidades sobre técnica, preparación física y táctica del fútbol, sino que ha de saber aplicar conocimientos sobre psicología deportiva para gestionar eficazmente el equipo en su singularidad.

En la actualidad está creciendo el apoyo gubernamental y federativo para la adecuada formación educativa de los entrenadores (Duffy et al, 2011), pese a que, como Findlay y Corbett (2002) denuncian, existe el agravio comparativo con otras profesiones que cuentan con un derecho a la práctica en exclusiva. Un entrenador no está reconocido como tal; sin embargo, está sometido a las responsabilidades propias de una profesión, máxime en su papel como formadores educativos de niños en edad escolar. 


\section{LA INTERVENCIÓN SOBRE EL TRIÁNGULO FORMATIVO: FORMADOR, NIÑO Y FAMILIAS.}

La unidad mínima que integra el esquema de la triangulación interpersonal formador-niño-familia (figura 2) se considera la base sobre la que cimienta el escenario de forma habitual (Gimeno, 2000; Sánchez, 2001), incluso contemplando la recurrente posibilidad de que un propio padre ejerza como entrenado. La figura de un padre-entrenador puede facilitar la adherencia al deporte o generar una presión adicional para el niño, derivado de unas sobre-expectativas y la difícil tarea de ser ecuánime para diferenciar entre exigencias deportivas y personales-familiares, que también puede conllevar una disociación de roles al adulto (Weiss \& Fretwell, 2005; Buceta, 2015). Uno de los retos deriva de la entrada prematura de jóvenes entrenadores y familiares de los deportistas como entrenadores de niños (Duffy et al, 2011).

Tradicionalmente, se ha evidenciado la necesidad de atender a los tres referentes para describir e intervenir sobre la cultura que se crea en este escenario, por lo que existen diversos intentos por educar, no sólo a los niños, sino también atender al comportamiento de los familiares mediante programas específicos y pautas de comportamiento para la sensibilización paterna o 'entrenamiento a padres' (Gimeno, 2000; Hernández, 2005; Garrido, Campos \& Castañeda, 2010; Prat \& Ventura, 2010; Diputación Foral de Bizkaia, 2012; Colegio Oficial de Psicólogos de Castilla La Macha, 2013; Jasso et al, 2013). Las recomendaciones instan a una delimitación de áreas de intervención entre familias, que han de suponer un acompañamiento emocional incondicional con evaluaciones constructivas, y los entrenadores, a quienes se les concede una responsabilidad para la coordinación del equipo como formadores deportivos, educadores y mediadores sociales. De este modo, se persigue un entorno adulto coherente, sin solapamientos ni contradicciones: están "condenados a entenderse (por el bien de los chicos)" en favor de la calidad formativa de los jóvenes deportistas (Buceta, 2015, p. 241).

La frecuencia de comportamientos que se consideran escasamente ejemplarizantes para los niños caricaturizan el escenario a nivel público (Arufe, 2015) y motiva que se dinamicen un gran número de intervenciones, a modo de pautas o decálogos, que quedan exhibidas en los espacios en los que se desarrolla el deporte escolar a modo de prevención.

FIGURA 2

Triángulo formativo sobre el que tradicionalmente se ha intervenido en el fútbol base.

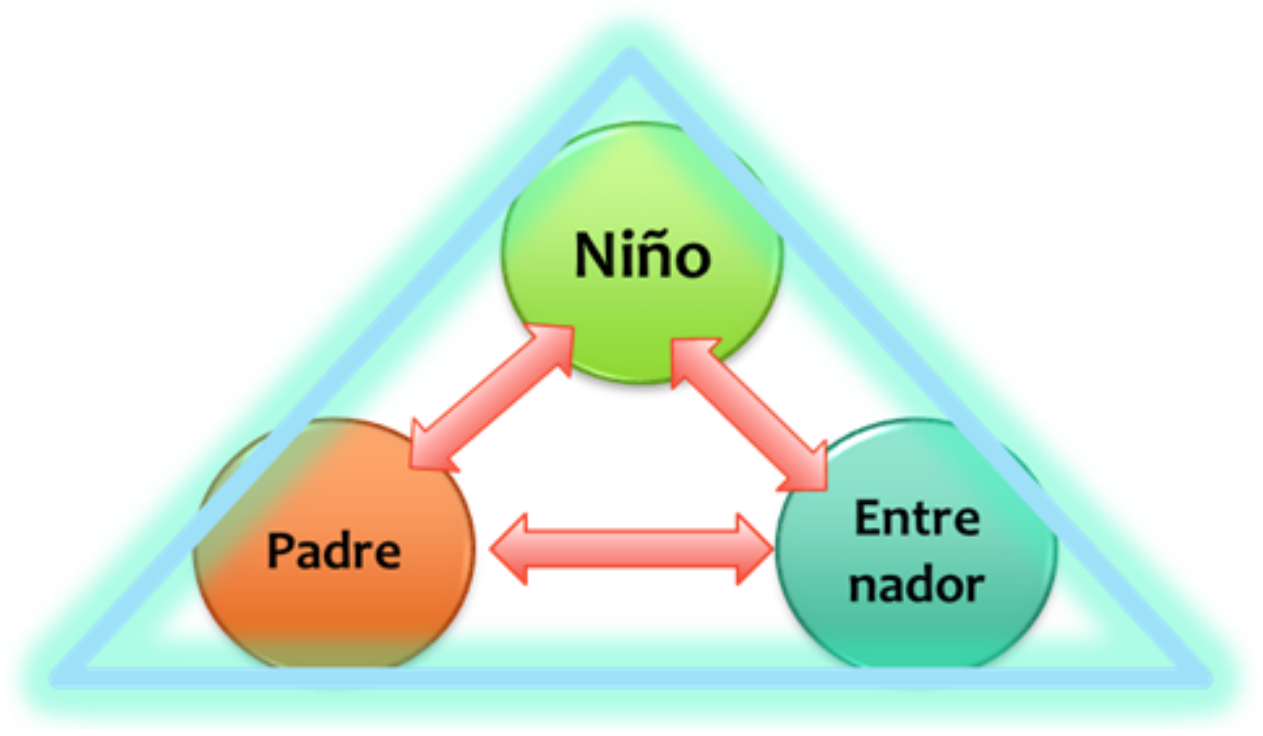

Fuente: elaboración propia. 
Existen estudios que constatan cómo el esfuerzo por el desarrollo de estrategias coordinadas por el adulto que promocionan los valores que facilita el deporte en edad escolar, mediante programas de intervención, desembocan en una inherente capitalización por parte de quien los practica (García-Calvo, Sánchez-Oliva, Sánchez-Miguel, Leo \& Amado, 2012; Fuller, Percy, Bruening \& Cotrufo, 2013; Jiménez, López-Pastor \& Manrique, 2014; Ruiz, Ponce de Leon, Sanz \& Valdemoros, 2015; Veroz, Yagüe \& Tabernero, 2015). Existe unanimidad en prescribir coherencia, cooperación y comunicación entre el entorno familiares y formadores por promover en positivo los valores que puede vehiculizar el deporte escolar y, en concreto, el fútbol.

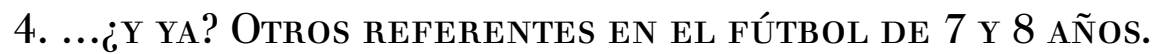

La literatura especializada da cuenta de la multireferencialidad intrínseca al escenario deportivoeducativo, refrendando el aforismo de que por sí solo, este triángulo no explica el fenómeno ocurrido en el escenario, sino que se trata de un entramado de relaciones intersubjetivas complejo y multirreferencial (Ardoino, 1993). Por ello, se parte de la revisión disciplinar vertebradora en tanto que se asume que es el adulto quien organiza cualquier actividad del niño, considerando que "se debe comprender que, en realidad, uno es con relación al otro. No se puede definir solamente de modo intrínseco ${ }^{2}$ " (Vallejo-Gomez, 2008, p. 251), es decir, el escenario es un todo, un tejido que no se puede descontextualizar para su estudio.

Cruz, Boixadós, Torregrosa y Mimbrero (1996) consideran que la encrucijada de relaciones en la que se ve inmerso el niño viene determinada: en primer lugar, por los principales agentes de socialización, padres, entrenadores y organizadores de la competición deportiva; en segundo lugar, por las diferentes condiciones contextuales (cuándo, dónde, con quién...), incluyendo elementos que superan el triángulo de formadorniño-familias; precisamente, hacia el que se ha invertido esfuerzos proponiendo pautas de comportamiento deseables (Gimeno, 2000; Sánchez, 2001). Además, es relevante la relación con sus pares, con quienes puede construir un aprendizaje tan o más significativo que lo pretendido en las programaciones de los entrenamientos (Lapresa, Arana \& Ponce de León, 1999). Del mismo modo, no puede obviarse la influencia del bagaje intrínseco que cada persona participante tiene.

Los medios de comunicación tienen un fuerte impacto en el niño y Veroz (2015) llega a contemplarlo como una variable contaminante del escenario socio-educativo. Justifica que las imágenes que se codifican a través de las pantallas transmites valores que confrontan con el juego limpio: juego violento o declaraciones cínicas para tratar de obtener rendimiento competitivo. El fútbol se convierte en un fenómeno social y comercial del 'deporte escaparate' (Lapresa et al., 2008). La televisión emite imágenes del deporte de alto rendimiento editadas en las que priorizan las situaciones polémicas, extradeportivas o resultadistas filtrándolas como de interés cultural (Avelaneda \& Lopes, 2012; Veroz, 2015). La filtración del deporte de élite es manifiesta en el fútbol base y predispone hacia la naturalización de la cultura de la violencia (Calzada, 2004). Si bien, las nuevas plataformas digitales y redes sociales han permitido que los departamentos de comunicación visibilicen la profundidad de las etapas formativas de fútbol, que utilizando plataformas como Facebook, Twitter, Instagram y YouTube muestran la instantaneidad de la realidad formativa, pudiendo implementar píldoras informativas y formativas de los miembros de los equipos, algo que favorece la permeabilidad y transparencia de las instituciones deportivas (Lobillo, 2018).

Incluso el uso de los videojuegos influye en la construcción del escenario, pues los nativos digitales desarrollan estrategias de aprendizaje autónomo con los videojuegos que más utilizan -entre los que se incluyen los de fútbol-. Se subraya que es posible aprovechar un uso educativo a través de las posibilidades motivacionales de los videojuegos gracias a su usabilidad y estética para el desarrollo de conceptos tácticos como el fuera de juego, el pase al hueco u otros (Armenteros, 2007; Pérez, 2014).

En cuanto a los organizadores de las competiciones, no sólo tienen impacto los implicados directos con el fútbol 'under 8' o 'sub-8': administración o federaciones, sino que las Asociaciones de Madres y Padres de Alumnos (AMPAs) proveen al escenario de una clave interpretativa esencial, dado que se erigen como 
copartícipes en la discriminación de actividades y asociaciones-empresas que tienen acceso a los alumnos de los colegios. Asimismo, es relevante la selección de los coordinadores de estas actividades, ya que sobre ellos recae la responsabilidad formativa de aportar un sentido saludable del deporte (Prat \& Ventura, 2010; Segarra, 2013). Los organizadores de las competiciones aportan las estructuras sobre las que el niño se educa, por lo que su incorporación al escenario es inexorable (Sánchez, 2014). En este escenario, los intereses formativo-deportivos y los económicos se disuelven en una ecuación mutuamente dependiente. Los cargos directivos de los equipos emergen como figuras clave en la comprensión de la identidad del equipo, pues han de gestionar el plano sistémico del club complementario al sentido formativo-deportivo de la actividad que gestionan. Estas dos claves, gestión sistémica del club y sentido formativo-deportivo, aportarán el capital económico y simbólico que les posicione en el campo deportivo (Bourdieu, 2003; Montero, 2013), que se traducirá, en términos coloquiales, en una reputación o reconocimiento social.

Asimismo, Prat, Flores y Carbonero (2013) indican que el papel del árbitro puede ser definitivo para la promoción de valores como la deportividad y el juego limpio según enfoque su intervención en el juego. Especialmente en el fútbol base, se le recomienda un sentido formativo y ético de su actuación, pese a que, por naturaleza, el comportamiento del árbitro se emprenda desde la coerción y la punición. En definitiva, son numerosos los elementos que confluyen en el escenario y todos ellos relevantes en su construcción y desarrollo.

Finalmente, se ha de considerar que de un modo institucional se concibe la formación físico-deportiva como la confluencia de la educación física, que se desarrolla en el colegio, y la actividad física desarrollada de modo libre como "complemento indispensable de la anterior" (Pastor, 2002, p. 16), por lo que implícitamente se reclama una comunicación entre el ambiente formal y no formal que establezca una pertinencia y adecuación de los aprendizajes que se promueven entre los alumnos/jóvenes deportistas para su práctica física habitual enfocada hacia la calidad de vida (Vizuete, 2013). La confluencia de todos estos referentes desemboca en la construcción de un espacio educativo y deportivo dinámico y complejo (figura 3). Los aprendizajes que se construyan derivan de la microcultura que cada equipo de fútbol base configura con las relaciones intersubjetivas que vehiculizan el sentido y significado atribuido a la actividad (Sabirón, 2006).

\section{FIGURA 3}

Esquema sintético del escenario de fútbol correspondiente a 7 y 8 años tras un análisis pormenorizado de todos los referentes que intervienen en la formación del niño.

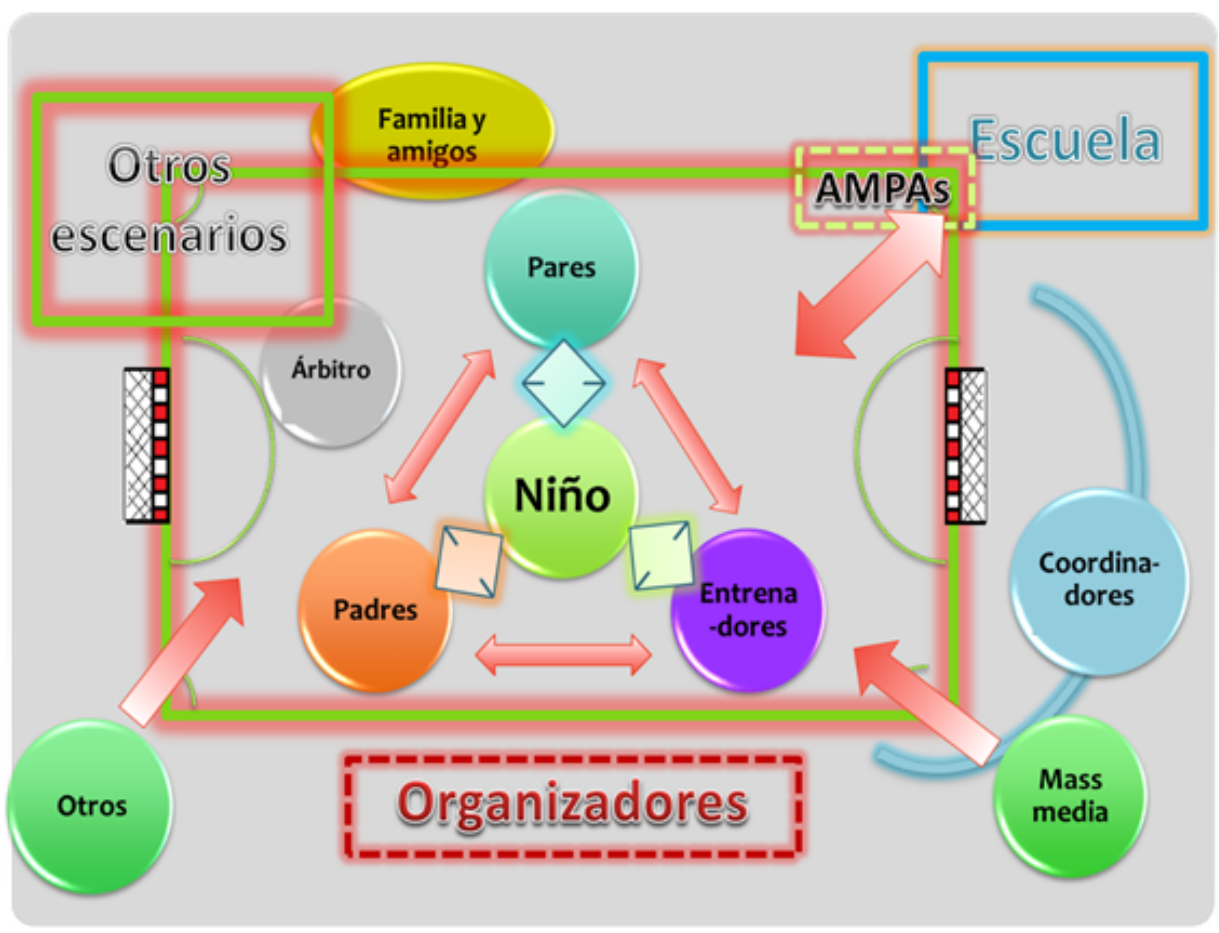

Fuente: elaboración propia. 


\section{Conclusiones}

El fútbol base se erige como un espacio socioeducativo en el que los niños pueden construir aprendizajes cualitativamente tan significativos como en los ambientes formales promovidos desde la escuela. La no formalidad del entorno favorece la permeabilidad de numerosos referentes que han de considerarse a la hora de emprender dinámicas formativas en el escenario. Esta premisa ha de considerarse a la hora de emprender cualquier intervención, pues el reduccionismo del escenario al esqueleto formado por padres, entrenadores y niños obvia claves interpretativas fundamentales en la construcción de la personalidad deportiva de los jugadores.

La falta de un análisis de partida que integre todos los referentes coexistentes en el fútbol motiva que la realidad formativo-deportiva actual continúe evidenciada por una gran falta de cohesión interna. Persiste una falta de coordinación entre las diferentes actividades que se dinamizan en el estado, derivado de su descentralización organizativa (Federación Española de Municipios y Provincias, 1992). Actualmente, existen varias propuestas potenciadoras del valor educativo del fútbol base como 'Diversala' -en Segovia-, 'Futgolines' -en Madrid- y 'Juga Verd Play' -en el Bajo Llobregat de Cataluña-, que priorizan la promoción de cuestiones educativas $^{3}$; en particular, la valoración positiva de las acciones de deportividad sobre el resultado competitivo, la adaptación de la normativa para favorecer la participación o la implicación del entorno. Sin embargo, se promueven de modo descontextualizado. Se precisa una reflexión asociacionista en común sobre estas prácticas formativo-deportivas a fin de esclarecer el objeto de estudio y fundamentar la pertinencia de una intervención deliberada hacia el valor educativo del deporte, en un ejercicio de interés formativodeportivo conjunto.

El estudio realizado refuerza la necesidad de desarrollar un modelo de deporte escolar emancipado del deporte de alto rendimiento. Se aprovecha la coyuntura de la competición reglada para incluir referentes como el alto rendimiento, el valor de la victoria o la comercialización del deporte. Si bien, se confirma que la situación de no formalidad facilita la incorporación de diversos agentes educativos (familia, entrenador y grupo de iguales), de referentes emocionales y axiológicos potenciadores de un aprendizaje efectivo para la vida. Resulta de suma relevancia no obviar las contradicciones en los procesos instructivos, el ejercicio de autoridad de los adultos o el protagonismo del niño en el equipo, al construir el escenario.

Las familias adquieren un rol de suma relevancia. El fútbol de niños de 7 y 8 años no sería posible en los mismos términos sin su implicación. No sólo acompañan a los niños en la preparación de la actividad y animan durante los partidos, sino que colaboran de un modo desinteresado activamente en el equipo de fútbol de sus hijos, pudiendo llegar a desempeñar cargos directivos, formativos o facilitando la organización del equipo. La implicación de las familias facilita la optimización de los recursos humanos en los clubes y, además, pagan las cuotas correspondientes, por lo que, sistémicamente, juegan un papel imprescindible en la organización de los equipos.

Asimismo, el formador y responsables de los equipos emergen como coordinadores de un escenario complejo, quienes ha de ampliar su rol instructivo-deportivo con otras funciones como la mediación social y el acompañamiento educativo. Aflora la necesidad explícita de una formación ajustada a las necesidades que debe atender en su ejercicio profesional: desarrollo de competencias para ejercer un papel mediador en los escenarios formativos-deportivos, que son construidos por todos los participantes, y adquisición de claves psico-socio-pedagógicas para gestionar la educación de sus jugadores prebenjamines en un entorno no formal; máxime sobre el devenir de la competición, que impregna algunos juicios evaluativos que vierten sobre sus hijos y/o jugadores. Se recomienda cierto tutelaje para que el adulto proporcione una evaluación constructiva atribuida al esfuerzo y aprendizaje del niño que redunde en una construcción efectiva de su autoconcepto. 
En definitiva, los adultos que promueven y hacen viable el desarrollo de la competición reglada como escenario socioeducativo a edades tan tempranas, han de garantizar que el referente educativo sobresalga en su identidad respecto al eje competitivo. Escuchar la voz de los niños, haciéndose eco de todos sus objetivos (disfrute en procesos y tiempos), proporciona interesantes oportunidades para acometer la empresa. Este trabajo revaloriza el entorno adulto como mediador de la formación del niño en un deporte que no educa por sí mismo, sino que ofrece unas excelentes oportunidades formativas que pueden optimizarse respecto al ejercicio actual y que ha de contar con todos los referentes del escenario.

\section{REFERENCIAS BIBLIOGRÁFICAS}

Abad, M.T., Giménez, F.J., Robles, J. \& Rodríguez, J.M. (2011). Perfil, experiencia y métodos de enseñanza de los entrenadores de jóvenes futbolistas en la provincia de Huelva. Retos. Nuevas tendencias en Educación Física, Deporte y Recreación, 20, 21-25.

Abós, A., Sevil, J., Sanz, M., Aibar, A. y García, L. (2015). Experiencias positivas en Educación Física a través de la intervención docente en el contenido de fútbol sala. Sportis Scientific Technical Journal, 1(5), 220-238.

Anderssen, N. \& Wold, B. (1992). Parental and peer influences on leisure-time physical activity in young adolescents. Research Quarterly for exercise and Sport, 63(4), 341-348.

Ardoino, J. (1993). L'approche multiréférentielle (plurielle) des situations éducatives et formatives. Pratiques de Formation, 25/26, 15-34.

Armenteros, M. (2007). Aprendizaje de las reglas del fútbol a través del videojuego. Comunicación y pedagogía: Nuevas tecnologias y recursos didácticos, 217, $42-47$.

Arufe-Giráldez, V.; Barcala-Furelos, R. y Mateos-Padorno, C. (2017). Programas de deporte escolar en España e implicación de los agentes educativos. Revista Internacional de Medicina y Ciencias de la Actividad Física y el Deporte. 17(67), 397-411.

Arufe, V. (2015). Cómo mejorar nuestra praxis de educadores deportivos. Una mirada hacia nosotros y hacia nuestros compañeros. En Arufe Giráldez, V. (Coord). V Congreso Mundial del Deporte Escolar, A Coruña, 5-7 noviembre 2015 (pp.1-12). A Coruña: Sportis.

Avelaneda, M. \& Lopes, C. (2012). El deporte y la televisión: El papel del profesional de Educación Física. Retos. Nuevas tendencias en Educación Física, Deporte y Recreación, 22, 33-37.

Bourdieu, P. (2003). Cuestiones de sociología. Madrid: Itsmo.

Buceta, J.M. (2015). Mi hijo es el mejor, y además es mi hijo. Manual de cabecera para padres de deportistas jóvenes. Madrid: Dykinson.

Calzada, A. (2004). Deporte y educación. Revista de Educación, 335, 45-60.

Cara, J.F., Pernía, I. \& Utrilla, M. (2014). Relaciones socio-afectivas entre jugadores, padres, entrenadores y árbitros en los campos de fútbol base. E-motion. Revista de Educación Motricidad e Investigación, 2, 78-87.

Castejón, F. J. (2008). Deporte escolar y competición. En I. Hernández, y F. Martínez (Coords.). El deporte escolar en la sociedad contemporánea. (pp. 159-177). Almería: Universidad de Almería.

Colegio Oficial de Psicólogos de Castilla La Mancha (2013). Guia de Orientación Psicológica para padres y educadores Deporte escolar. Albacete: Diputación de Albacete.

Cruz, J., Boixadós, A., Torregrosa \& Mimbrero, J. (1996). ¿Existe un deporte educativo?: papel de las competiciones deportivas en el proceso de socialización del niño. Revista de psicología del deporte, 4, 111-132.

Diputación Foral de Bizkaia (2012). Claves del deporte escolar para padres y madres. Recuperado de: https://www.biz kaia.net/kultura/kirolak/heziketa/index.asp\#texto (Última visita: 7 de Enero de 2017). 
Duarte, D., Garganta, J. \& Ferreira, A.M. (2014). Does the experience influence the efficacy of football coach? A perspective from coaches with different levels of experience as player and as coach. Journal of Human Sport and Exercise, 9(1), 17-30.

Duffy, P., Hartley, H., Bales, J., Crespo, M., Dick, F., Vardham, D... Curado, J. (2011). Sport coaching as a 'profession': Challenges and future directions. International Journal of Coaching Science, 5(2), 93-123. Recuperado de https: //www.coach.ca/files/Duffy_et_al_2011.docx (Última visita: 7 de Enero de 2017).

Federación Española de Municipios y Provincias (1992). Manifiesto sobre el deporte en la edad escolar. En Actas de las VII Jornada de deporte y corporaciones locales, El deporte en edad escolar, A Coruña, Octubre.

Findlay, H. \& Corbett, R. (2002). The key components of a profession. Coaches Report, 8(3), 25. Recuperado de http:/ /www.crdsc-sdrcc.ca/eng/documents/Knowing-the-law_Winter02_E.pdf(Última visita: 7 de Enero de 2017).

Fuller, R.D., Percy, V.E., Bruening, J.E. \& Cotrufo, R.J. (2013). Positive Youth Development: Minority Male Participation in a Sport-Based Afterschool Program in an Urban Environment. Research Quarterly for Exercise and Sport, 84 (4), 469-482.

García-Calvo, T., Sánchez-Oliva, D., Sánchez-Miguel, P. A., Leo, F. M. \& Amado, D. (2012). Escuela del deporte: Valoración de una campaña para la promoción de valores. Motricidad. European Journal of Human Movement, $28,67-81$.

Garrido, M.E., Campos, M.C. \& Castañeda, C. (2010). Importancia de los padres y madres en la competición deportiva de sus hijos. Revista Fuentes, 19, 173-194.

Garrido, M.E.; González, G. \& Romero, S. (2010). The behavior of parents in Municipals Sports Schools of Sevilla. Journal of Sport Health Research, 2 (3), 261-276.

Gilbert, W. \& Rangeon, S. (2011). Current directions in coaching research. Revista Iberoamericana de Psicología del Ejercicio y Deporte. 6(2), 217-236.

Gimeno, F. (2000). Entrenando a padres y madres... Claves para una gestión eficaz de la relación con los padres y las madres de jóvenes deportistas. Zaragoza: Gobierno de Aragón.

González, M. (2012). Evaluación del funcionamiento del primer curso de implantación de un Programa Integral de Deporte Escolar en Educación Primaria en el municipio de Segovia (Tesis Doctoral). Segovia: Universidad de Valladolid.

Hernández, E. (2005). Guía para padres: Deporte Escolar. Valencia: Ayuntamiento de Valencia. Fundación Deportiva Municipal.

Ibáñez, S., del Barrio, J. Gómez, C. \& Araujo, C. (2007). Crónica del Plan Escolar. Madrid: Consejo Superior de Deportes.

Isorna, M., Ruiz, F. y Rial, A. (2013). Variables predictoras del abandono de la práctica físico-deportiva en adolescentes. Cultura, ciencia y deporte: revista de ciencias de la actividad fisica y del deporte de la Universidad Católica de San Antonio, 23, 93-102.

Jasso, J., Almanza, C.R., Conchas, M., Rivero, J., Paredes, R. \& Jiménez, D. J. (2013). Estrategia de promoción de valores a través del fútbol en la escuela «Frida Kahlo» de la ciudad. Retos: nuevas tendencias en educación física, deporte y recreación, 24, 105-106.

Jiménez, B., López-Pastor, V.M., \& Manrique, J.C. (2014). Evaluación comparativa de resultados de un programa municipal de deporte escolar. Retos: Nuevas Tendencias en Educación Física, Deporte y Recreación, 26, 15-20.

Lapresa, D., Arana, J., Garzón, B., Egüén, R. \& Amatria, M. (2008). Enseñando a jugar “el fútbol”. Hacia una iniciación coherente. Logroño: Universidad de La Rioja y Federación Riojana de Fútbol.

Lapresa, D., Arana, J. \& Ponce de León, A. (1999). Orientaciones educativas para el desarrollo del deporte escolar. Logroño: Universidad de La Rioja y Federación Riojana de Fútbol.

Lledó, E., Martínez, G. \& Huertas, F. (2014). Perfil del entrenador de fútbol en la etapa escolar en escuelas de clubes de élite de la Comunitat Valenciana. Cultura, ciencia y deporte: Revista de Ciencias de la Actividad Fisica y del Deporte de la Universidad Católica de San Antonio, 25, 57-68.

Llopis, D. (2011). Cualidades de un buen entrenador de fútbol. Abfutbol: revista técnica especializada en fútbol, 49, 81-87. 
Lobillo, G. (2018). Desarrollo comunicacional en los clubes de fútbol españoles. Estudio de caso: Sevilla FC. Revista Iberoamericana de Ciencias de la Actividad Física y el Deporte, 7(1), 34-46.

López, J.A., (2005). El desarrollo metacognitivo y su relación con el aprendizaje escolar. Recuperado de: https://www.c op.es/colegiados/T-00921/metacog-infant.pdf (Última visita: 7 de Enero de 2017).

Merino, A., Arraiz, A, \& Sabirón, F. (2016). Family involvement in under 7s school football in La Rioja: interests, motivations, roles and family dynamics. Sportis Scientific Technical Journal, 2 (1), $58 \# 76$.

Merino, A., Arraiz, A, \& Sabirón, F. (2017a). Procesos formativos en el fútbol prebenjamín: El paso de la ingenuidad a la institucionalización. Educación Fisica y Ciencia, 18(2), e013.

Merino, A., Arraiz, A, \& Sabirón, F. (2017b). Estudio descriptivo sobre el entrenador de fútbol sala prebenjamín en La Rioja: perfil, motivaciones, cualidades y dificultades. Retos: Nuevas Tendencias en Educación Física, Deporte y Recreación, 31, 164-170.

Merino, A., Sabirón, F. \& Arraiz, A. (2015). Análisis del escenario de competición en fútbol prebenjamín: Un estudio de caso. Retos: Nuevas Tendencias en Educación Física, Deporte y Recreación, 28, 26-32.

Monjas, R., Ponce, A. \& Gea, J.M. (2015). La transmisión de valores a través del deporte. Deporte escolar y deporte federado: relaciones, puentes y posibles trasferencias. Retos: Nuevas Tendencias en Educación Física, Deporte y Recreación, 28, 276-284.

Montero, A. (2013). Dinámicas familiares y procesos de socialización en el deporte en edad escolar (Tesis Doctoral). Universidade da Coruña, La Coruña.

Nuviala, A., León, J.A., Gálvez, J. \& Fernández, A. (2007). Qué actividades deportivas escolares queremos. Qué técnicos tenemos. Revista Internacional de Medicina y Ciencias de la Actividad Física y el Deporte, 25, 1-9.

Ossorio, D. (2012). Deporte, contexto social y familia la cuestión de las actitudes de los padres. Lecturas: Educación fisica y deportes, 165. Disponible en: http://www.efdeportes.com/efd165/deporte-contexto-social-y-familia.ht m (Última visita: 7 de Enero de 2017).

París, F. (2015). La práctica deportiva en España. En III Congreso de la Federación de Asociaciones de Gestores del Deporte de España. Deporte: Una Cuestión de Estado, Santander.

Pastor, J.L. (2002a). Aproximación histórica a la evolución de la Educación Física en España (1883-1990). Historia de la educación: Revista interuniversitaria, 21, 199-214.

Pérez, A. (2014). El aprendizaje con videojuegos. Experiencias y buenas prácticas realizadas en las aulas españolas. Escuela Abierta, 17, 135-156

Piéron, M. (2002). Estudi sobre els hảbits esportius dels escolars d'Andorra. Andorra: Ministeri d'Educació Joventut i Esports.

Prat, M., Flores, G. \& Carbonero, L. (2013). El rol del árbitro y su implicación en el fomento del juego limpio. Análisis y propuestas de intervención en el contexto de deporte universitario. Retos. Nuevas tendencias en Educación Física, Deporte y Recreación, 24, 72-78.

Prat, M. \& Ventura, C. (2010). Deportes y educación en valores. Dirigido a madres y padres interesados por el deporte escolar. Madrid: Confederación Española de Asociaciones de Padres y Madres de Alumnos.

Raudsepp, L. \& Viira, R. (2000). Sociocultural correlates of physical activity in adolescents. Pediatric Exercise Science, 12, 51-60.

Reschly, A. M. y Christenson, S.I. (2009). Parents as Essential Partners for Fostering Students' Learning Outcomes. En R. Gilman, E. Scott, y M. Fourlong (Coords.). Handbook of Positive Psychology in Schools. (pp. 257-272). New York: Taylor \& Francis.

Romero, S. Garrido, M.E. y Zagalaz, M.L. (2009). El comportamiento de los padres en el deporte. Retos. Nuevas Tendencias en Educación Física, Deporte y Recreación, 15, 29-34.

Ruiz, J.V., Ponce de León, A., Sanz, E. \& Valdemoros, M.A. (2015). La educación en valores desde el deporte: investigación sobre la aplicación de un programa integral en deportes de equipo. Retos: Nuevas tendencias en educación física, deporte y recreación, 28, 270-276. 
Sánchez, D.L. (2001). Influencia de la familia en el deporte escolar. Revista Digital EFDeportes, 40. Disponible en htt p://www.efdeportes.com/efd40/familia.htm (Último visita 7 de enero de 2017).

Sánchez, J. (2014). Análisis del desarrollo del juego del fútbol, desde la óptica de los sistemas complejos (Tesis doctoral). Las Palmas de Gran Canaria: Universidad de las Palmas de Gran Canaria.

Sabirón, F. (2006). Métodos de investigación etnográfica en Ciencias Sociales. Zaragoza: Mira.

Segarra, E. (2013). El deporte en escolar. En E. Segarra, A. García de Alcaraz, E. Ortega \& A. Díaz (Eds.). Deporte en Edad Escolar: Alternativas y modelos organizativos (pp.5-12). Murcia: Universidad de Murcia.

Suldo, S.M. (2009). Parent-Child Relationships. En R. Gilman, E. Scott, y M. Fourlong (Coords.). Handbook of Positive Psychology in Schools. (pp. 245-265). New York: Taylor \& Francis.

Vallejo-Gomez, N. (2008). La pensée complexe: Antidote pour les pensées uniques, Entretien avec Edgar Morin. Synergies Monde, 4, 249-262.

Veroz, R. (2015). Análisis comparativo del desarrollo de valores socio-educativos en competición de fútbol en iniciación (Tesis Doctoral). León: Universidad de León.

Veroz, R., Yagüe, J.M. y Tabernero, B. (2015). Incidencia de dos modelos de competición de fútbol sobre los valores socio-educativos en prebenjamines. Retos. Nuevastendencias en Educación Física, Deporte y Recreación, 28, 84-89.

Vizuete, M. (2013). El Deporte en la edad escolar en la sociedad de los nativos digitales. Necesidad de un cambio de paradigma. En E. Segarra, A. García de Alcaraz, E. Ortega y A. Díaz (Eds.). Deporte en Edad Escolar. Alternativas y modelos organizativos (pp. 13-48). Murcia: Universidad de Murcia.

Weiss, M. R. \& Fretwell, S.D. (2005). The Parent-Coach/Child-Athlete Relationship in Youth Sport: Cordial, Contentious, or Conundrum? Research Quarterly for Exercise and Sport, 76 (3), 286-303.

\section{Notas}

1 Constructo propuesto por Castejón (2008) para tratar de trazar una imagen mental de la organización espacial de familias y allegados que envuelve al fenómeno del deporte escolar en los días de competición. En este estudio sería transferible a su concreción 'futbolcentrista'.

2 La cita textual pertenece a Edgar Morin durante su entrevista con N. Vallejo-Gomez (2008) en la que se profundiza sobre el pensamiento complejo.

3 El desarrollo de las propuestas formativas se puede seguir en: https://www.deportivoindependiente.es/, "Diversala”, ht tps://www.futgolines.com/, "Futgolines” y https://cebllob.org/, "Juga Verd Play". 